OPEN ACCESS

Edited by:

Peter Dovc

University of Ljubljana, Slovenia

Reviewed by:

Adam John Watkins,

University of Nottingham,

United Kingdom

Jian Xu,

Chinese Academy of Fishery

Sciences (CAFS), China

*Correspondence:

Linyuan Shen

shenlinyuan0815@163.com

Lianqiang Che

clianqiang@hotmail.com

Li Zhu

zhuli7508@163.com

tThese authors have contributed equally to this work

Specialty section: This article was submitted to

Livestock Genomics,

a section of the journa

Frontiers in Genetics

Received: 09 March 2018

Accepted: 12 July 2018

Published: 15 August 2018

Citation:

Shen L, Gan M, Zhang S, Ma J, Tang G, Jiang Y, Li M, Wang J, Li X,

Che L and Zhu L (2018)

Transcriptome Analyses Reveal Adult Metabolic Syndrome With Intrauterine

Growth Restriction in Pig Models.

Front. Genet. 9:291.

doi: 10.3389/fgene.2018.00291

\section{Transcriptome Analyses Reveal Adult Metabolic Syndrome With Intrauterine Growth Restriction in Pig Models}

\author{
Linyuan Shen ${ }^{1,2 * t}$, Mailin Gan ${ }^{1 t}$, Shunhua Zhang ${ }^{1}$, Jideng Ma1', Guoqing Tang', \\ Yanzhi Jiang ${ }^{3}$, Mingzhou $\mathrm{Li}^{1}$, Jinyong Wang ${ }^{4}$, Xuewei $\mathrm{Li}^{1}$, Lianqiang Che ${ }^{5 *}$ and Li Zhu ${ }^{1 *}$ \\ ${ }^{1}$ College of Animal Science and Technology, Sichuan Agricultural University, Chengdu, China, ${ }^{2}$ Department of Cell \\ and Developmental Biology, University of Illinois at Urbana-Champaign, Champaign, IL, United States, ${ }^{3}$ College of Life \\ Science, Sichuan Agricultural University, Chengdu, China, ${ }^{4}$ Chongqing Academy of Animal Science, Chongqing, China, \\ ${ }^{5}$ Institute of Animal Nutrition, Sichuan Agricultural University, Chengdu, China
}

Epidemiological data have indicated that intrauterine growth retardation (IUGR) is a risk factor for the adult metabolic syndrome in pigs. However, the causative genetic mechanism leading to the phenotype in adulthood has not been well characterized. In the present study, both normal and IUGR adult pigs were used as models to survey the differences in global gene expression in livers through transcriptome sequencing. The transcriptome libraries generated $104.54 \mathrm{gb}$ of data. In normal and IUGR pigs, 16,948 and 17,078 genes were expressed, respectively. A total of 1,322 differentially expressed genes (DEGs) were identified. Enrichment analysis of the DEGs revealed that the top overrepresented gene ontology (GO) terms and pathways were related to oxidoreductase activity, ATPase activity, amino catabolic process, glucose metabolism, and insulin signaling pathway. The increased gluconeogenesis (GNG) and decreased glycogen synthesis in the liver contributed to the glucose intolerance observed in IUGR. The reduced expression of insulin signaling genes (such as PI3K and AKT) indicated an elevated risk of diabetes in adulthood. Together, these findings provide a comprehensive understanding of the molecular mechanisms of adult IUGR pigs and valuable information for future studies of therapeutic intervention in IUGR metabolic syndrome.

Keywords: pig, mRNA, transcriptome, IUGR, liver, gluconeogenesis

\section{INTRODUCTION}

The period of intrauterine growth and development is one of the most vulnerable periods in mammalian lifetime. Unfortunately, impairments to the embryo/fetus or its organs during gestation result in intrauterine growth restriction (IUGR) (Verma and Chaudhary, 2016). In humans, about $23.8 \%$ of newborns and approximately 30 million babies worldwide suffer from IUGR every year (Harmayani et al., 2017). Previous studies have associated increased 
morbidity and mortality, delayed postnatal growth and development, and increased susceptibility to metabolic syndrome with IUGR early in life (Pallotto and Kilbride, 2006). As the number of cases with metabolic syndromes is rapidly increasing, fetuses and neonates with IUGR are strongly predisposed to obesity, diabetes, and cardiovascular disease in later life based on experimental animal models and human epidemiological data (McMillen and Robinson, 2005; Manten et al., 2007; Martin-Gronert and Ozanne, 2007). The liver is the most important organ in the regulations of numerous metabolic processes, including hormone production and glucose and lipid metabolism (Ramayo-Caldas et al., 2012). Until now, hepatic metabolism is the preferential model system through which mechanisms of IUGR are examined. For example, offsprings exposed to intrauterine malnutrition have an increased risk of developing abnormal glucose metabolism along with increased hepatic gluconeogenesis (Thorn et al., 2009, 2013; George et al., 2012) and reduced hepatic glycolysis (Bogdarina et al., 2004). Additionally, defective insulin signaling cascade pathway would be expected to impair glucose uptake in the muscle, which would induce overproduction of glucose in the liver, ultimately leading to the development of glucose intolerance (Bogdarina et al., 2004; Samuel and Shulman, 2012). It has also been reported that IUGR pigs showed increased fatty acid flux toward the liver and reduced lipolysis and fatty acid oxidation (Yan et al., 2017). Together, these changes can lead to lipotoxicity, further reducing insulin sensitivity in the liver (Karpe et al., 2011; Yan et al., 2017). Recently, hepatic mitochondrial dysfunction was demonstrated to be a potential mechanism of IUGR development. These studies reported that IUGR during infancy decreased the capacity of hepatic mitochondrial biogenesis and the metabolism efficiency of oxidative phosphorylation (OXPHOS), resulting in excess production of mitochondrial superoxide radicals and poor hepatic antioxidant defense systems (Che et al., 2015; Zhang et al., 2016, 2017).

Although there is a wealth of evidence to show that IUGR can increase the risk of adult metabolic diseases such as cardiovascular disease, obesity, and type 2 diabetes (Ross and Beall, 2008; Rinaudo and Wang, 2012), the genetic mechanisms underlying IUGR are not well characterized. At present, nextgeneration sequencing, a powerful method that can be used to understand complete gene regulatory networks relevant to IUGR, is being applied to analyze global transcriptomic changes (Mutz et al., 2013). Although there are some studies about transcriptome of IUGR organs (such as placenta, kidney, etc.) in human and mouse models (Tain et al., 2015; Ackerman et al., 2017), metabolic syndrome of IUGR induced by aberrant liver transcriptome is still not well characterized. Therefore, we used pigs as biomedical models for the study of the metabolic syndrome of IUGR in humans because of the similar morphology, physiology, metabolism, and proportional organ sizes between pigs and humans. Furthermore, pigs have the advantage of relatively low genetic and environmental variances, whereas humans live in a highly confounding environment, where personal lifestyle choices such as smoking and alcohol consumption have significant impacts on the incidence of metabolic syndrome ( $\mathrm{Li}$ et al., 2012). The aim of this study was to employ pig hepatic transcriptome analyses to reveal the genetic mechanisms of metabolic syndrome arising from IUGR.

\section{MATERIALS AND METHODS}

\section{Ethics Statement}

All the animal experimental and sample collection procedures were approved by the Institutional Animal Care and Use Committee of the College of Animal Science and Technology of Sichuan Agricultural University, Sichuan, China, under permit No. DKY-B20131403 (Ministry of Science and Technology, China, revised in June 2004). All experimental methods were performed in accordance with the Sichuan Agricultural University of Health Guide for the Care and Use of Laboratory Animals.

\section{Animal Housing and Sample Collection}

A total of eight pairs of normal birth weight $(\sim 1.49 \mathrm{~kg})$ and IUGR $(\sim 0.97 \mathrm{~kg})$ PIC breeding male piglets chosen from eight sows were used in this study. One IUGR piglet and one control piglet were selected from each litter. All selected pigs were moved to nursing cages at 7 days of age. The animals were fed with milk formula until 28 days of age. The formula composition and nutrient levels of the milk formula fed to the pigs are provided in Supplementary Table S1. After 28 days, the composition of diets fed to pigs was adjusted according to body weight (Supplementary Table S2). In all stages, pigs had ad libitum access to feed and water and were housed in the same environment. At 150 days of age, all pigs were kept off feed but given free access to water for $24 \mathrm{~h}$, then electrically stunned, exsanguinated, scalded, and rinsed. Serum was harvested by centrifugation at $3000 \mathrm{~g}$ for $10 \mathrm{~min}$ at $4^{\circ} \mathrm{C}$ and stored at $-80^{\circ} \mathrm{C}$. Other samples were obtained from the core of the liver and longissimus dorsi immediately after exsanguination and rapidly frozen in liquid nitrogen.

\section{Biochemical Parameters and Metabolite Measurement}

The concentrations of glucose (No. F006), glucogen (No. A043), triglyceride (\#A110-2), free fatty acid (No. A042-1), and malondialdehyde (MDA) (No. A003-1) were determined using commercial kits (Nanjing Jiancheng Institute of Bioengineering, Nanjing, Jiangsu, China) following the manufacturer's instructions. The levels of hepatic adenosine triphosphate (ATP) and adenosine diphosphate (ADP) were measured using high-performance liquid chromatography (HPLC), following a previously published method (Zhang et al., 2017). The activity of ATPase (No. A070-2), cytoplasmic copper/zinc superoxide dismutase (CuZn SOD) (No. A062), and glutathione reductase (GR) was also determined using commercial kits (Nanjing Jiancheng Institute of Bioengineering, Nanjing, Jiangsu, China). Sample data were collected using an automatic biochemical 
analyzer (Model 7020, Hitachi, Tokyo, Japan) and a UV-1100 spectrophotometer (Shanghai Mapada Instruments Co. Ltd., Shanghai, China).

\section{Intravenous Glucose Tolerance Test}

After overnight fasting, an intravenous glucose tolerance test (i.v.GTT) was conducted in all animals as previously described by Wang et al. (2016). Briefly, at 149 days of age, dextrose (500 g/L) was infused continuously through ear venipuncture over the course of $6 \mathrm{~min}$. The infusion dose and rate were $0.5 \mathrm{~g} / \mathrm{kg}$ of body weight and $10 \mathrm{~g}$ glucose/minute, respectively. Blood samples were obtained at $-6,-4,-2$, and $0 \mathrm{~min}$ relative to the completion of dextrose infusion and at 5, 10, 15, 30, 60, 90, and $120 \mathrm{~min}$ post administration. Blood glucose was measured using an Ascensia Elite glucometer (Bayer Healthcare Company, Leverkusen, Germany). All samples were measured in duplicate.

\section{Total Ribonucleic Acid (RNA) and Deoxyribonucleic Acid (DNA) Extraction}

Total RNA was extracted from liver and muscle samples using TRIzol (Invitrogen, CA, United States). Nucleic acids were then further purified with RNeasy column (Qiagen, United States) according to the manufacturer's protocol. RNA integrity and concentration were assessed using the Bioanalyzer 2100 (Agilent Technologies) and NanoDrop (Thermo Technologies), respectively. DNA was isolated from liver samples for the measurement of mitochondrial DNA (mtDNA) copy number using the DNeasy Blood \& Tissue Kit (Qiagen, United States).

\section{Measurement of mtDNA Copy Number}

The relative mtDNA copy number was determined by quantitative polymerase chain reaction (qPCR). The quantitative reverse transcription polymerase chain reaction (qRT-PCR) was performed using the SYBR Green Real-time PCR Master Mix (Takara, Dalian, China) on a CF96 Real-Time PCR Detection System (Bio-Rad, Richmond, CA, United States). The ratio of mitochondrial genes (ATP6 and COX2) to nuclear DNA single copy gene (GCG) within the same sample was used to calculate the mtDNA content (primer sequences are listed in Supplementary Table S3). All reactions were performed in triplicate. Relative mtDNA copy number per diploid cell was calculated by the $2^{\Delta C t}$ method.

\section{Messenger RNA (mRNA) Library Construction and Sequencing}

We randomly selected three pigs from each group for deep sequencing analysis. First, only RNA samples that had RNA Integrity Number (RIN) scores $>8$ were used for sequencing. Approximately $5 \mu \mathrm{g}$ of total RNA from a single liver tissue was isolated by binding of the Poly (A) tails on the mRNA with poly-T oligo conjugated magnetic beads (Thermo Fisher). Following purification, the mRNA was fragmented into small pieces using divalent cations under elevated temperature. The cleaved RNA fragments were then reverse transcribed into the final complementary DNA (cDNA) library in accordance with the Illumina RNA ligation-based method (Illumina, San
Diego, CA, United States). The constructed RNA libraries were sequenced using an Illumina HiSeq 2000 platform. Paired-end reads, approximately 90 bp long, were generated.

\section{Transcriptome Data Analysis}

Clean data were obtained by removing reads containing adapters, reads containing over $10 \%$ of poly $(\mathrm{N})$, and low-quality reads ( $>50 \%$ of the bases had Phred quality scores $\leq 10$ ) from the raw data. All the downstream analyses were conducted only on high-quality data. The index of the reference genome was built using Bowtie v2.0.6 and paired-end clean reads were aligned to the reference genome using TopHat v2.0.14. The mapped reads from each library were assembled with Cufflinks v2.2.1. The reference annotation-based transcript (RABT) assembly method in Cufflinks v2.2.1 was used to construct and identify mRNA transcripts from the TopHat2 alignment results. Cuffdiff v2.1.1 was used to calculate fragments per kilobase of exon model per million mapped reads (FPKM) scores for transcripts in each library. Differentially expressed genes (DEGs) were identified through pairwise comparisons between every two stages using edgeR (release 3.2). The statistical significance of gene expression differences was evaluated using an adjusted $P$-value $(Q$-value $)<0.01$ and $\mid \log _{2}$ (fold change) $\mid \geq 2$ as the threshold. In addition, the cluster of the DEGs was performed by using common perl and R scripts.

\section{Functional Enrichment Analysis}

Differentially expressed genes were converted to human orthologous genes and submitted to the DAVID 6.8 web server ${ }^{1}$ for enrichment analysis of the significant overrepresentation of GO biological processes (GO-BP), molecular function (GO-MF) terminologies, and Kyoto Encyclopedia of Genes and Genomes (KEGG) pathway category. In all tests, the $P$-values were calculated using Benjamini-corrected modified Fisher's exact test. Only $P$-values $<0.05$ were considered as significant.

\section{Quantitative RT-PCR}

The expression levels of selected genes were quantified using qRT-PCR. The qRT-PCR was performed using the SYBR Green Real-time PCR Master Mix (Takara, Dalian, China) on a CF96 real-time PCR detection system (Bio-Rad, Richmond, CA, United States). The PCR primer sequences used are presented in Supplementary Table S3. $A C T B, T B P$, and $T O P 2 B$ genes were simultaneously used as internal control genes for normalization. All measurements contained a negative control (no cDNA template). Each RNA sample was analyzed in triplicate. The $2^{-\Delta \Delta C t}$ method was used to determine the relative abundance of each mRNA.

\section{Statistical Analyses}

Data were analyzed with SPSS (21.0 version). All data were presented as means \pm standard deviation (SD). Differences in groups were analyzed with Student's $t$-test. $P<0.05$ was considered to be statistically significant.

\footnotetext{
${ }^{1}$ https://david.ncifcrf.gov/
} 


\section{RESULTS AND DISCUSSION}

\section{Phenotypic Traits of Adult IUGR Pig}

According to a previous study (Che et al., 2010), pigs with birth weights within 0.5 standard deviations of the mean litter birth weight were considered to be normal, whereas those with lower weights (1.5 standard deviations below the mean litter weight) were defined as IUGR. As presented in Figure 1A, IUGR piglets had lower birth weights $(32-39 \%$ decrease, $p<0.01)$ than normal piglets, and this difference persisted until adulthood (19-25\% decrease, $p<0.01)$. This suggests that impaired intrauterine growth and development may have a persistent influence on metabolic efficiency later in life. It was observed that adult IUGR pigs exhibited greater plasma concentrations of glucose $(p<0.01)$ and lower hepatic glycogen $(p<0.05)$ (Figures 1B,C). Previous studies have reported that hyperglycemia phenotypes are typically associated with glucose intolerance and type 2 diabetes (Nathan et al., 2009). These findings were supported by our observations during the intravenous glucose tolerance test (Figure 1D). Blood glucose concentrations were higher at postinfusion times $5(p<0.05)$ and $10 \mathrm{~min}(p<0.05)$ in IUGR pigs than normal group. Moreover, adult IUGR pigs also exhibited greater plasma concentrations of triglyceride and free fatty acids, as well as triglyceride content in livers (Figures $\mathbf{1 B}, \mathbf{C}$ ). These results imply that IUGR likely impairs the capacity of hepatic fatty acid oxidation, thus promoting the development of the hyperlipidemia phenotype. This is consistent with recent studies reporting that IUGR was the predominant factor in affecting lipid metabolism (Koklu et al., 2006; Li et al., 2016). Interestingly, the influence of high circulating triacylglycerol and non-esterified fatty acid was also demonstrated to promote glucose intolerance (Karpe et al., 2011; Lopez et al., 2011). Furthermore, OXPHOS was determined to be an important component of hepatic metabolism. This was consistent with other previous studies, which demonstrated that suckling piglets of IUGR impaired hepatic mitochondrial biogenesis and energy homeostasis (Zhang et al., 2017). As shown in Figures 1E,F, mtDNA and ATP were both significantly decreased in adult IUGR pigs $(p<0.05)$. This implies that the change of the OXPHOS pathway during gestation might persist well into adult life. Overall, these results suggest that adult IUGR pigs have multiple metabolic syndromes, and differences in the transcriptome were likely to underlie the aberrant phenotypes.

\section{Summary of Transcriptome Data}

To identify genes involved in the differentially effective metabolic pathway between adult IUGR and normal pigs, RNA transcriptome libraries were constructed using adult pig liver tissues. Transcriptome libraries generated a total of 1,161 million pair-end reads of $90 \mathrm{bp}$ in length. The total sequencing length was $104.54 \mathrm{gb}$, representing approximately $43 \times$ coverage of the complete pig genome. Approximately $64.30-68.68 \%$ of all reads were unique and aligned to the University of California, Santa Cruz (UCSC) pig reference genome (Sus scrofa 11.1) using the TopHat2 package (Table 1). Furthermore, $85.84-91.41 \%$ of all reads were located within exons (Supplementary Figure
S1). In this study, if one transcript was expressed in all the three biological replicates of the constructed cDNA libraries, it was considered to be an expressed transcript and included for subsequent analysis. Consequently, 16,948 and 17,078 known transcripts were identified as being expressed in normal and IUGR pigs, respectively. Among the transcript populations, only a small number of genes were highly expressed (Supplementary Tables S4, S5). These findings were consistent with previous studies, which reported that only a small number of highly expressed genes may play cellular housekeeping gene roles and regulate cellular components and basal cellular metabolism (Shen et al., 2015). The reproducibility and reliability of transcriptome libraries were analyzed by total expressed genes using hierarchical clustering. As presented in Figure 2A, the three biological replicates of each group were highly correlated with one another (Pearson's $r>0.96$ ), and all the three libraries of each group were definitively assigned to a single cluster. This verified the high reproducibility and reliability of the transcriptome profiling performed in the present study. To explore the global transcriptional changes, we identified a total of 16,554 transcripts which were co-expressed in normal and IUGR pigs and identified 1322 DEGs (Figure 2B and Supplementary Table S6), including 558 highly expressed genes in the normal group and 764 highly expressed genes in the IUGR group (Figures 2C,D). The heat map of the hierarchical clustering analysis indicated that the DEG data were also highly reproducible (Figure 2C). Furthermore, the transcriptome sequencing results were also validated through the qRT-PCR analysis of expression patterns of eight randomly selected genes from each group. The results indicated that the expression patterns of these genes were highly consistent between the two methods (Pearson's $r>0.81, p<0.01$; Supplementary Figure S2).

\section{Functional Enrichment Analysis for Differential Expression Genes}

To explore the potential functions of DEGs, we performed a GO enrichment analysis. As shown in Figure 3A, the top significantly overrepresented GO terms were related to two main classes of biological functions: energy metabolism (including oxidation reduction process, oxidoreductase activity, ATP binding, and ATPase activity) and amino and glucose metabolism (including serine/threonine kinase activity, D-alanine and D-serine catabolic processes, and detection of glucose) (Supplementary Table S7). Mitochondrion is considered as the central organelle with respect to its functions in energy metabolisms and the major sites of ATP productions in the body (Hock and Kralli, 2009). Interestingly, previous studies have suggested that mitochondrial dysfunction is an underlying mechanism responsible for the IUGR. Mortensen et al. (2010) observed that mouse IUGR offsprings exhibited abnormal expression patterns of mitochondrial function-related genes in liver tissue. Also, in a juvenile pig model, it has been reported that post-weaning IUGR pigs exhibited impaired mitochondrial respiration functions, reduced mitochondrial DNA (mtDNA) contents and ATP productions, and impaired antioxidant defense systems in livers (Liu et al., 2012; Che et al., 2015; 

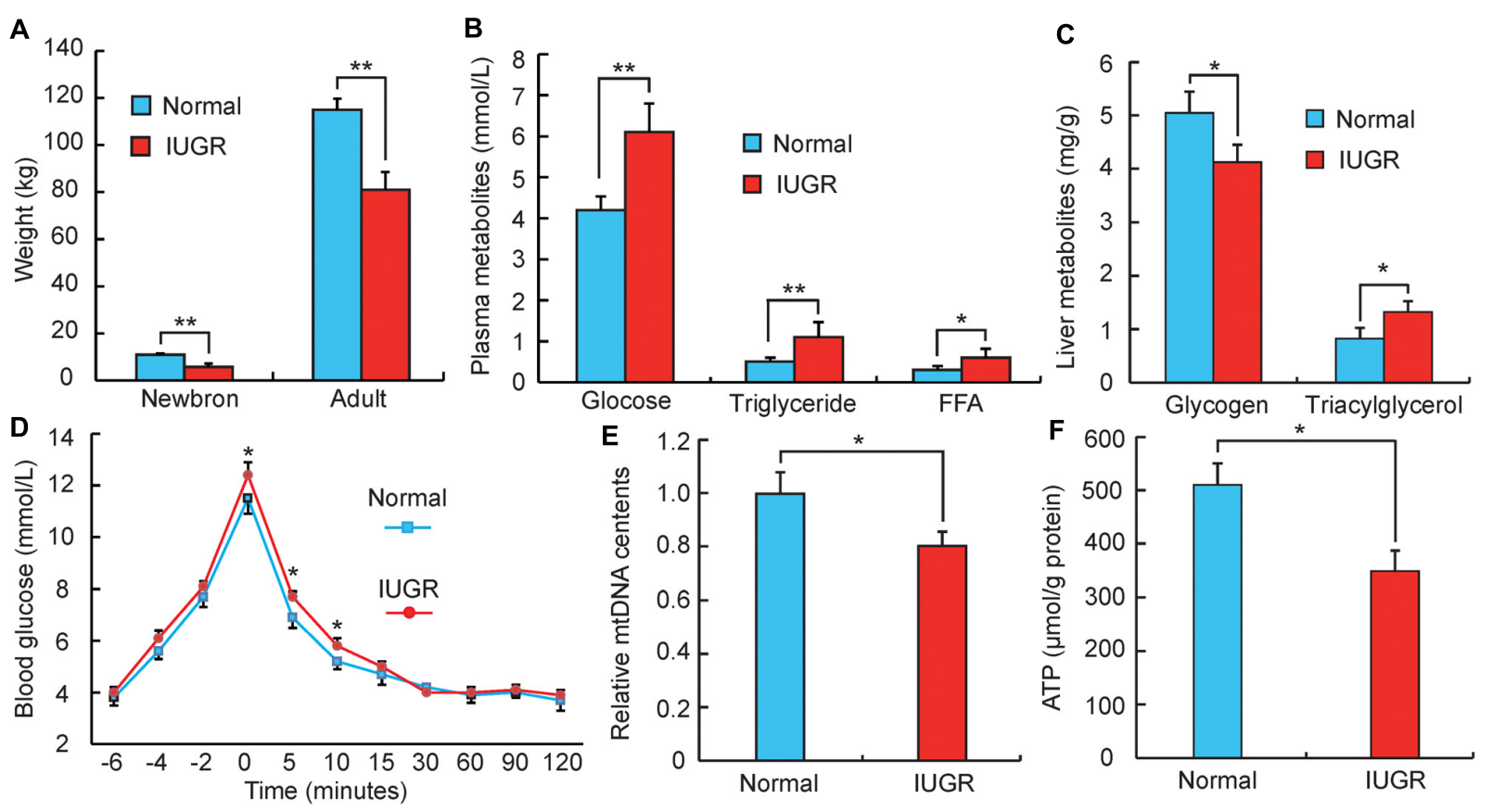

FIGURE 1 | Phenotypic differences between adult normal and IUGR pigs. (A) The body weights of normal and IUGR pigs as newborns and adults ( $n=8)$. (B) The blood glucose, triglyceride and free fatty acid (FFA) concentrations in adult normal and IUGR pigs $(n=8)$. (C) The contents of glycogen and triglyceride in livers of adult normal and IUGR pigs $(n=8)$. (D) Plasma glucose concentrations after an intravenous glucose tolerance test (i.v.GTT) at 149 days of age. Time indicates minutes relative to the completion of dextrose infusion $(n=8)$. (E) The copy number of hepatic mitochondrial DNA in adult normal and IUGR pigs $(n=8)$. (F) The contents of ATP in livers of adult normal and IUGR pigs $(n=8)$. Data are means \pm SD. Statistical significance was calculated by Student's $t$-test $(n=8$ per individual) Significant differences levels: ${ }^{*} p<0.05,{ }^{* *} p<0.01$.

Zhang et al., 2017). Moreover, amino acids are one of the major sources of carbon for hepatic gluconeogenesis, such as alanine, serine, and threonine (Sandoval and Sols, 1974; Egan et al., 1983). Here, we observed that serine and threonine catabolic metabolisms were highlighted in the functional enrichment analysis, which was in accordance with a previous report, in which IUGR status was associated with up-regulated gluconeogenic gene expressions (PEPCK, G6P, and PGC1 $\alpha$ ), which resulted in increased hepatic gluconeogenic capacity in a fetal sheep model (Thorn et al., 2009). Furthermore, previous studies have reported that elevated hepatic gluconeogenesis would reduce glucose tolerance (Thorn et al., 2011). These findings are consistent with our observations reported in Figure 1D.
Here, we also performed a KEGG pathway enrichment analysis. As presented in Figure 3B, the top enriched pathways were related to amino acid metabolisms (such as tryptophan, glycine, arginine, and ornithine), which are consistent with the activated pathway of hepatic gluconeogenesis in IUGR animals (Thorn et al., 2009). In addition, we also observed that pyruvate served as the substrate for gluconeogenesis, and this metabolic pathway was significantly enriched in IUGR animals (Seubert and Huth, 1965). The highlighted insulin signaling pathway and the development of type 2 diabetes mellitus in animals suggest that diabetes may be a fetal adaptation to undernutrition and likely persists until adulthood (Figure 3B and Supplementary Table S8). These findings are consistent with a previous report in which glucose intolerance and diabetes in pigs born from IUGR

TABLE 1 | Summary of transcriptome alignment.

\begin{tabular}{|c|c|c|c|c|c|c|}
\hline Group & Normal-1 & Normal-2 & Normal-3 & IUGR-1 & IUGR-2 & IUGR-3 \\
\hline \multirow[t]{2}{*}{ Total raw reads } & 182462984 & 188671562 & 188490354 & 212157764 & 191055696 & 198755512 \\
\hline & $(16.42 \mathrm{G})$ & $(16.98 G)$ & $(16.96 \mathrm{G})$ & $(19.09 \mathrm{G})$ & $(17.20 \mathrm{G})$ & $(17.89 G)$ \\
\hline Valid data & 181246976 & 186904702 & 186844562 & 210590742 & 189842370 & 197354938 \\
\hline Valid ratio (reads) & 0.99 & 0.99 & 0.99 & 0.99 & 0.99 & 0.99 \\
\hline Q20\% & 99.50 & 99.55 & 99.63 & 99.53 & 1.00 & 1.00 \\
\hline Total mapped ratio \% & 72.49 & 70.11 & 69.57 & 70.68 & 73.79 & 73.96 \\
\hline Unique mapped ratio \% & 66.90 & 64.32 & 64.30 & 65.61 & 68.56 & 68.68 \\
\hline GC content \% & 46.43 & 45.26 & 45.18 & 45.12 & 45.82 & 45.22 \\
\hline
\end{tabular}



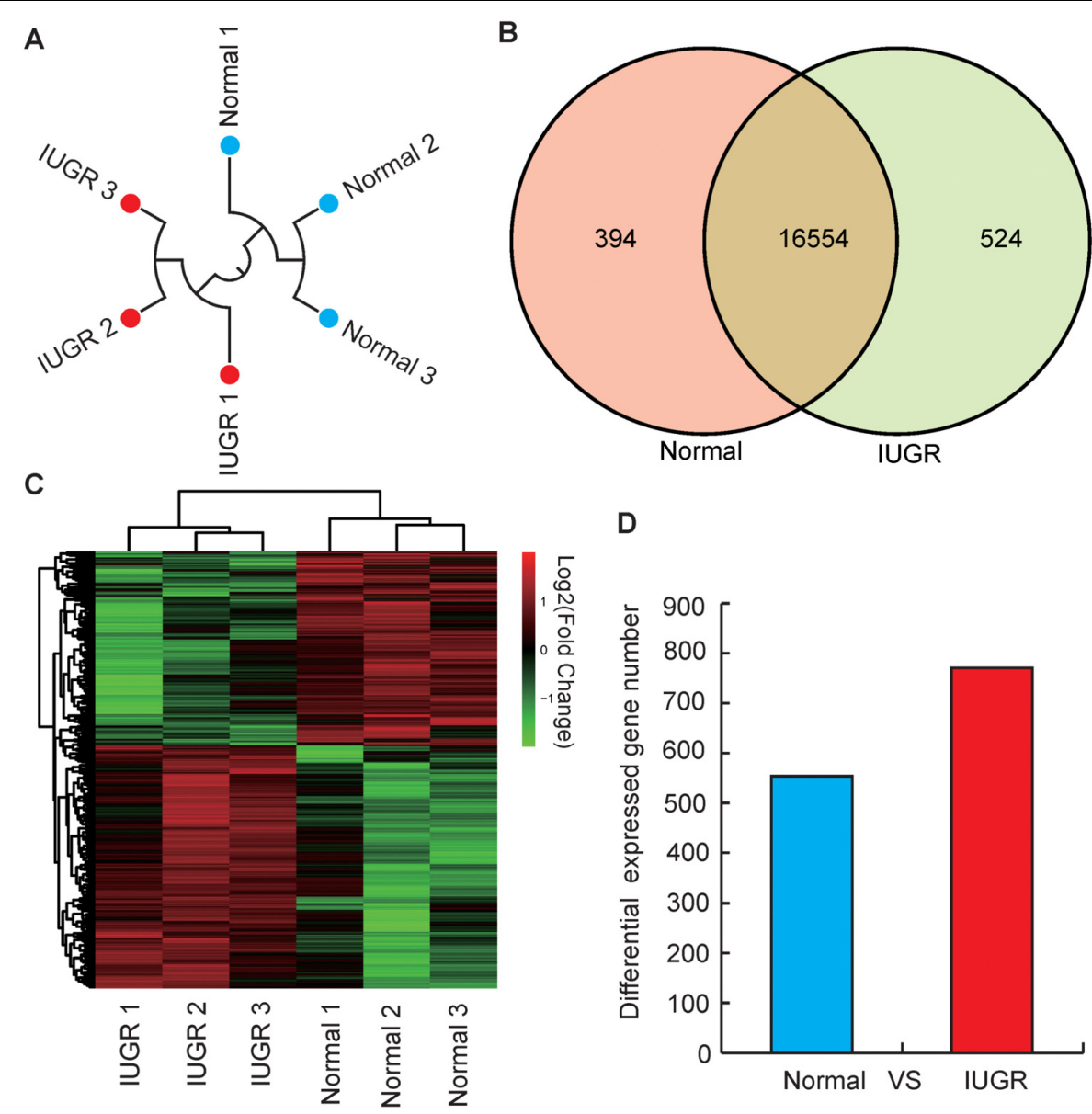

D

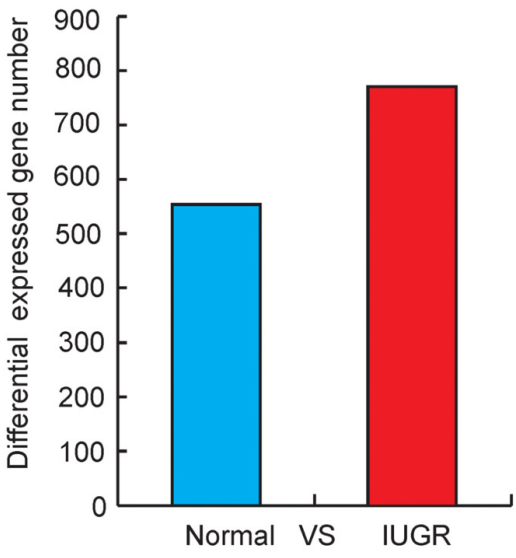

FIGURE 2 | Genome-wide distribution of DGEs between normal and IUGR pigs. (A) Hierarchical clustering of samples using all expressed genes for biological reproducibility analysis. (B) Venn diagram of expressed genes between normal and IUGR pigs. (C) Heat map diagram of DGEs between normal and IUGR pigs (D) Distribution of DEGs between normal and IUGR pigs.

conditions were associated with down-regulated expression of insulin signaling genes ( $I R, P I 3 K$, and $A K T 1 / 2)$ in the liver (Wang et al., 2016). As shown in Figure 3B, the peroxisome proliferatoractivated receptor (PPAR) signaling pathway was involved in the regulation of the AMPK-SIRT1-PGC1 $\alpha$-PPAR signaling cascade, which is responsible for mitochondrial biogenesis, fatty acid oxidation, and OXPHOS (Cheng and Ristow, 2013). Overall, the transcriptome data have indicated that IUGR has a strong impact on many metabolic pathways in adult pigs.

\section{Differential Expression of Genes Related to Glucose and Lipid Metabolism}

The excess accumulation of endogenous glucose played a crucial and direct role in the development of hyperglycemia and glucose intolerance. In the liver, the gluconeogenesis pathway is an important metabolic pathway for endogenous glucose synthesis, which is regulated by several important rate-limiting enzymes, such as PEPCK, G6PC, PC, and FBP1 (Wolfrum et al., 2003). Previous studies have demonstrated that increased gene expression of any of these enzymes would increase hepatic glucose production and plasma glucose levels in diabetics (Wolfrum et al., 2003; Collier and Scott, 2004). As presented in Figure 4A, the mRNA levels of PEPCK $(p<0.05)$, G6PC $(p<0.01)$, and $P C(p<0.05)$ were significantly up-regulated in liver tissues from IUGR pigs. In addition, FOXO1, a nuclear receptor relevant to glucose metabolism, was up-regulated in IUGR animals (Figure 4A). This results in the promotion of hepatic gluconeogenesis by directly binding to the PEPCK and G6PC promoters (Zhang et al., 2006; Jitrapakdee, 2012). Taken together, these results suggested that the capacity of hepatic gluconeogenesis is elevated in adult IUGR pigs. Glycogen synthesis in the liver is the alternate strategy through which endogenous glucose content is regulated. We observed that the expression of glycogen synthesis gene (GYS) was significantly decreased in IUGR pigs (Figure 4B), which was consistent with the observed glycogen levels in livers of IUGR pigs (Figure 1C). The skeletal muscle was another important tissue participating in the regulation of glucose metabolism, which could dispose of approximately $70-80 \%$ of postprandial glucose (Krook et al., 2000). GLUT4 is considered to be the rate-limiting step of insulin-induced glucose uptake into the muscle (Krook 


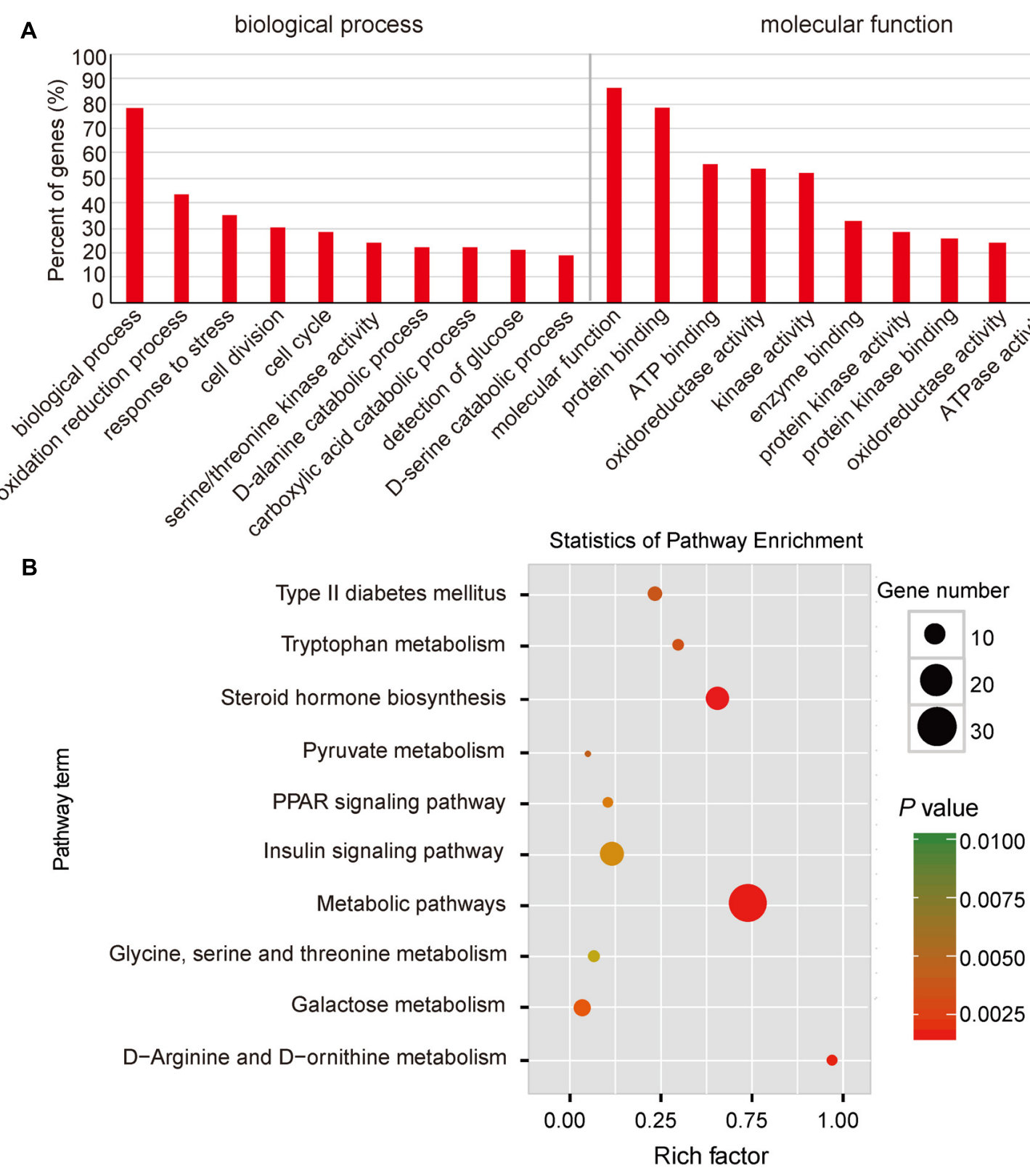

FIGURE 3 | Functional enrichment analysis of DGEs between normal and IUGR pigs. (A) Gene Ontology (GO) categories enriched for DEGs between normal and IUGR pigs. (B) KEGG pathway enrichment analysis of DGEs between normal and IUGR pigs. The P-values were calculated using Benjamini-corrected modified Fisher's exact test.

et al., 2000). Previous studies have reported that defection of GLUT4 could induce severe glucose tolerance (Kim et al., 2001), whereas overexpression of GLUT4 would be expected to improve insulin sensitivity (Leturque et al., 1996). As expected, our results indicated that GLUT4 expression in muscle tissue was significantly decreased in IUGR pigs (Figure 4C), which also agreed with the reduced glycogen content in muscle (Figure 4D). Overall, these outcomes contributed to hyperglycemia and glucose intolerance observed in adult IUGR pigs.

Insulin signaling is the major pathway to regulate glucose homeostasis, which is regulated by coordinating several important metabolic processes including glucose uptake, glycolysis, glucose oxidation, glycogen synthesis, and lipid synthesis and degradation in the liver (Saltiel and Kahn, 2001). The IRS-PI3K-AKT signaling cascade is the major insulin signaling pathway. Knockdown of expressions of those proteins would be expected to enhance gluconeogenesis and impair glycogen synthesis (Saltiel and Kahn, 2001). As expected, we observed that the mRNA levels of $P I 3 K$ and $A K T$ were decreased in livers from IUGR pigs (Figure 4E). Consequently, the decreased expression of AKT may further target GSK3 $\beta$ and activate its expression (Rommel et al., 2001), which would 

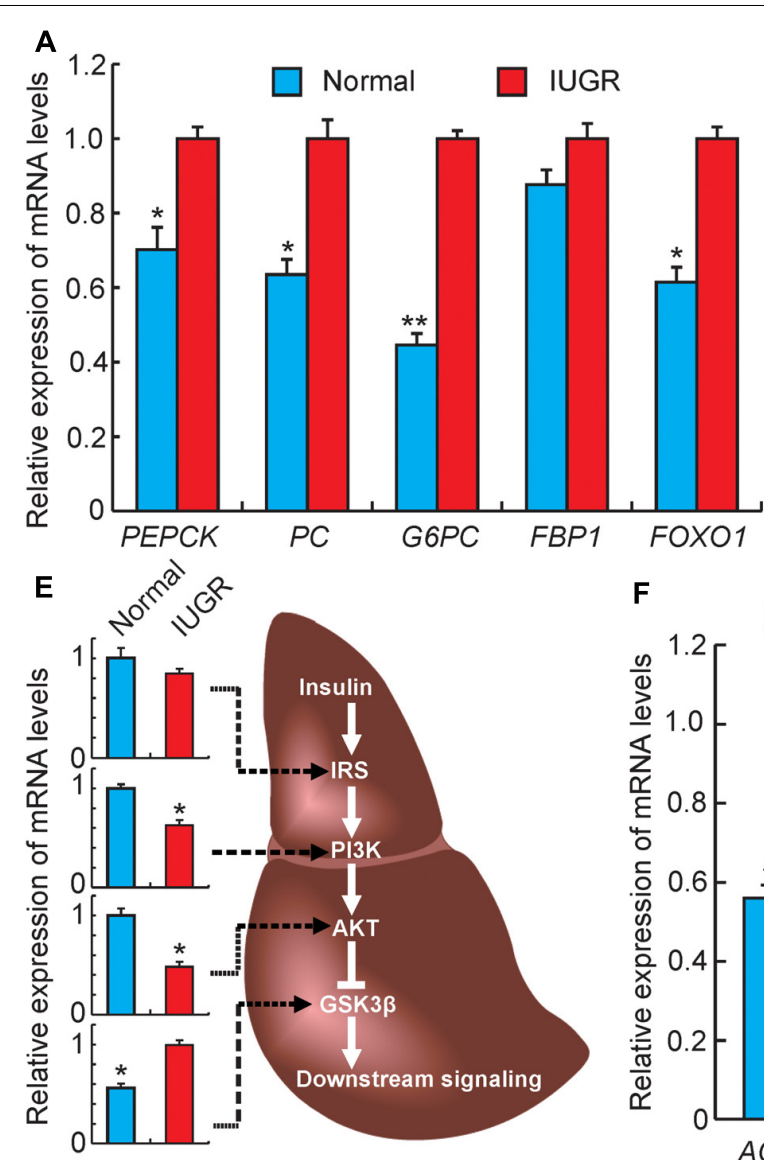

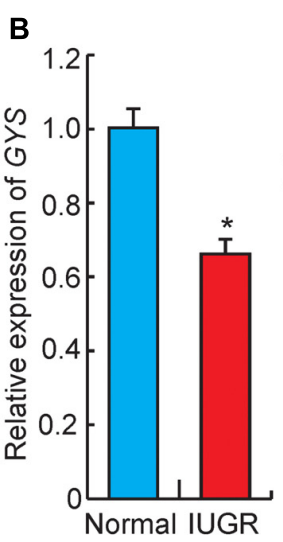

$\mathbf{F}$

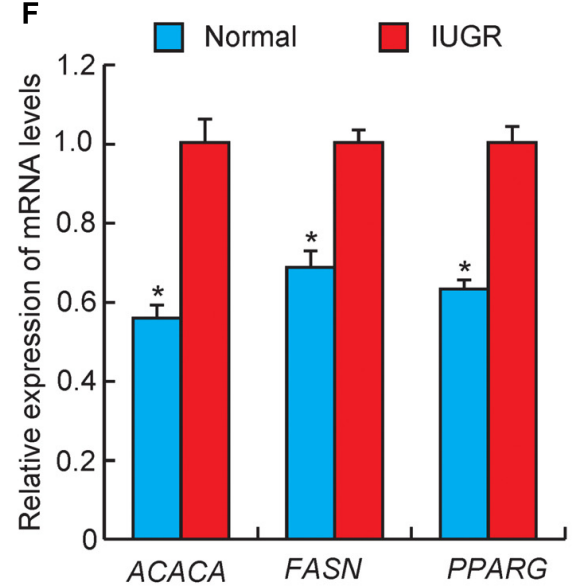

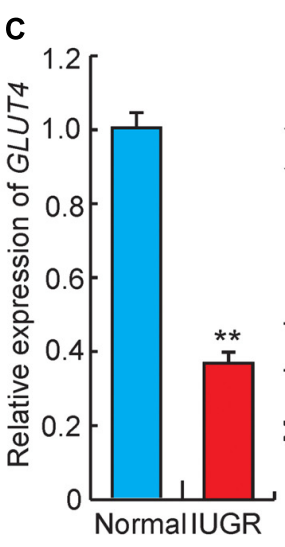

D

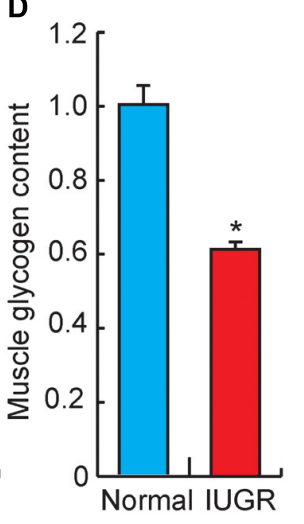

G

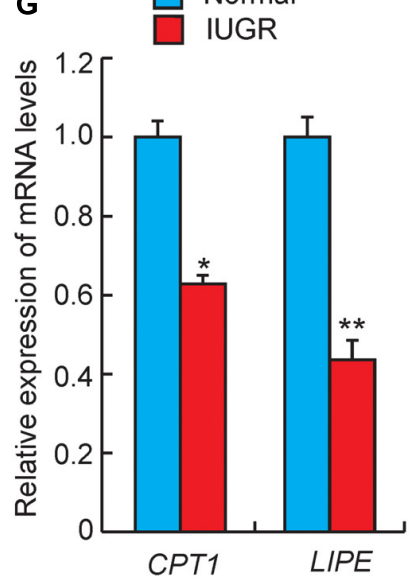

FIGURE 4 | Gene expression related to glucose and lipid metabolism. (A) The expression levels of gluconeogenesis rate-limiting enzymes genes in livers. PEPCK, phosphoenolpyruvate carboxykinase; PC, pyruvate carboxylase; G6PC, glucose-6-phosphatase; FBP1, fructose-1,6-bisphosphatase 1; FOXO1, forkhead box O1. (B) The expression levels of glycogen synthesis gene (GYS) in livers. (C) The expression levels of glucose transporter gene (GLUT4) in muscles. (D) The glycogen levels in muscles. (E) The expression levels of genes related to insulin signaling in livers. IRS, insulin receptor substrate; $P / 3 K$, phosphatidylinositol 3-kinase; $A K T$, serine/threonine protein kinases; GSK3 $\beta$, glycogen synthase kinase 3 beta. (F) The expression levels of fatty acid synthesis genes in livers. ACACA, acetyl-CoA carboxylase alpha; FASN, fatty acid synthase. (G) The expression levels of fatty acid oxidation genes in livers. CPT1, diacylglycerol cholinephosphotransferase; LIPE, lipase, hormone sensitive; PPARG, peroxisome proliferator activated receptor gamma. Data are means \pm SD. Statistical significance was calculated by one-way repeated-measures analysis of variance ( $n=3$ per individual). ${ }^{*} p<0.05,{ }^{* *} p<0.01$.

result in increased expression of gluconeogenic genes (such as FOXO1), accompanied by the promotion of gluconeogenesis and the impairment of glycogen synthesis (Sakamaki et al., 2012). This is similar to a previous study, which reported that maternal protein restriction would reduce the expression of insulin signaling proteins and lead to hyperinsulinemia in 21-monthold female rats (Fernandez-Twinn et al., 2005). Furthermore, insulin signaling was also involved in lipid metabolism, and a previous study has reported that the process of gluconeogenesis is correlated with increased fatty acid synthesis (Saltiel and Kahn, 2001). Nebendahl et al. (2013) reported that IUGR offsprings exhibited increased liver lipid contents than their littermates born with normal birth weights (Nebendahl et al., 2013). We also observed that IUGR pigs have higher triglyceride levels in livers than normal pigs (Figure 1C). The fatty acid synthesis genes (ACACA and FASN) and fat deposition gene (PPAR $\gamma)$ were also observed to be up-regulated in livers of IUGR pigs (Figure 4F). However, other studies have demonstrated that increased lipogenesis in non-adipose tissues of IUGR offsprings may be attributed to impaired fatty acid oxidation capacity. Therefore, to further explore the mechanism of fatty acid oxidation in IUGR, we assessed expression patterns of genes related to lipolysis. $C P T-1$ is the rate-limiting enzyme that determines fatty acid oxidation, and LIPE cleaves fatty acids from intracellular triglycerides for oxidation and export (Snel et al., 2012). As shown in Figure 4G, the expression levels of CPT-1 and LIPE were down-regulated in adult IUGR livers, suggesting that the capacity of fatty acid oxidation was decreased in livers of IUGR pigs.

\section{Differential Expression of Genes Related to Mitochondrial Biogenesis and Energy Metabolism}

At present, IUGR suppression of mitochondrial function has been demonstrated in suckling pigs (Zhang et al., 2016, 

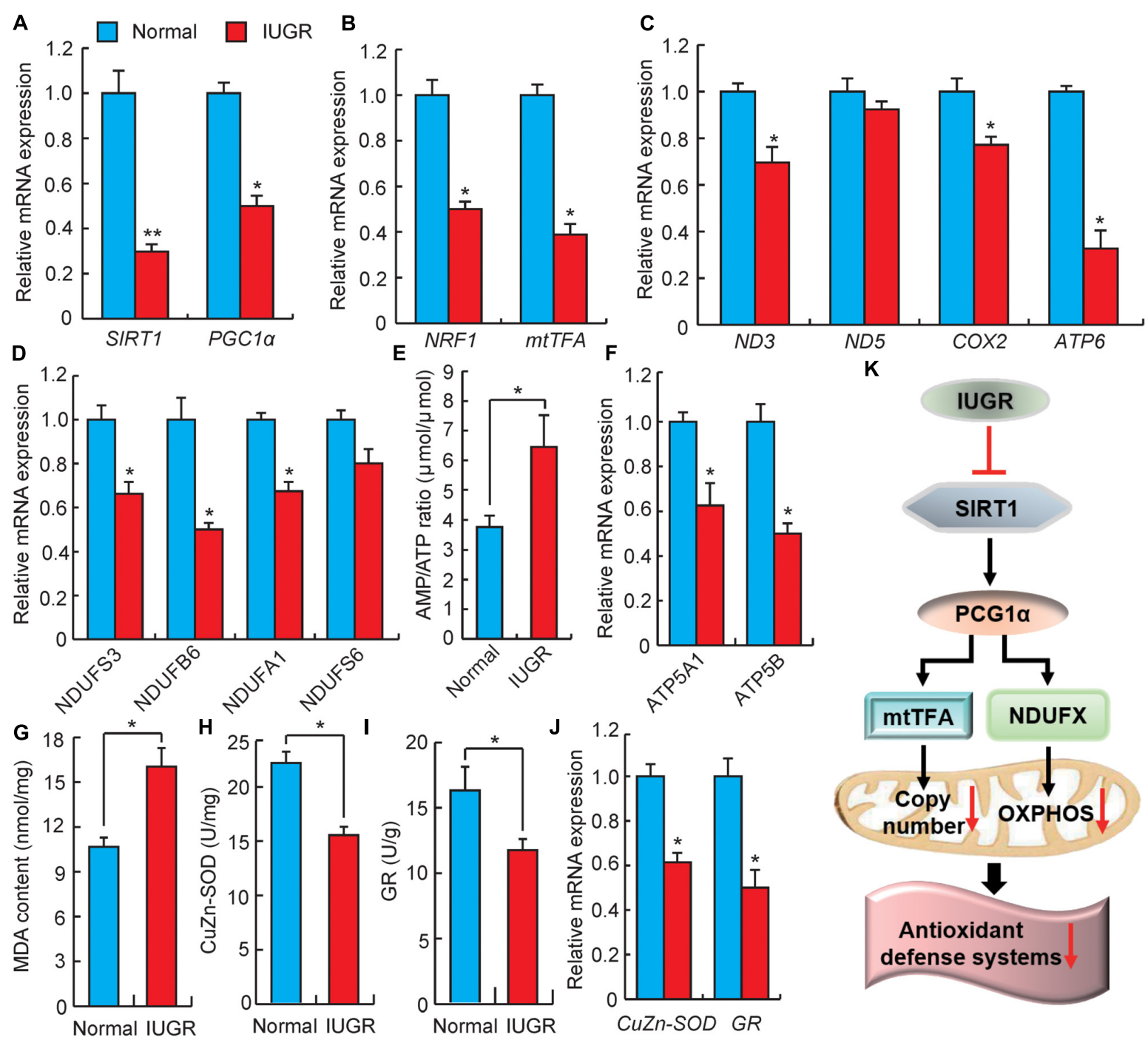

FIGURE 5 | Analysis of genes involved in mitochondrial biogenesis and energy metabolism. (A) DEGs involved in energy homeostasis. SIRT1, sirtuin 1; PGC1 $\alpha$, PPAR coactivator 1 alpha. (B) Expression levels of mitochondrial biogenesis related genes. NRF1, nuclear respiratory factor 1; mtTFA, transcription factor A, mitochondrial. (C) The relative expression levels of mitochondrial genomic genes. ND3, NADH dehydrogenase subunit 3; ND5, NADH dehydrogenase subunit 5; COX2, cytochrome c oxidase subunit II; ATP6, ATP synthase FO subunit 6. (D) The expression levels of genes associated with the electron transport chain. NDUF, NADH:ubiquinone oxidoreductase subunit. (E) The ratio of AMP/ATP in livers. (F) The expression levels of ATP synthesis genes. ATP5A1, ATP synthase, H+ transporting, mitochondrial $\mathrm{F} 1$ complex, alpha subunit 1; ATP5B, ATP synthase, $\mathrm{H}+$ transporting, mitochondrial $\mathrm{F} 1$ complex, beta polypeptide. (G) The contents of malondialdehyde (MDA) in livers. (H) The activity of CuZn-SOD in livers. (I) The activity of GR in livers. (J) The relative expressions of CuZn-SOD and GR genes. CuZn-SOD, Superoxide dismutase 1; GR, glutathione reductase. (K) Possible pathways of IUGR mediation of hepatic energy metabolism and oxidative stress in adulthood. Data are means \pm SD. Statistical significance was calculated by one-way repeated-measures analysis of variance $(n=3$ per individual). $* p<0.05$, ${ }^{* *} p<0.01$.

2017), but the mechanisms resulting in this phenomenon in adults remain to be thoroughly characterized. SIRT1 is considered to be a sensor of energy metabolism, which physically interacts with deacetylated PGC1 $\alpha$ at multiple lysine sites (Cantó and Auwerx, 2009). Increasing SIRT1 activity could modulate $P G C 1 \alpha$ functions in vivo and ultimately modulates the regulation of energy homeostasis (Lagouge et al., 2006). As shown in Figure $\mathbf{5 A}$, the expressions of SIRT1 and PGC1 $\alpha$ were significantly down-regulated in livers of adult IUGR pigs. $P G C 1 \alpha$ is the central regulatory factor mediating mitochondrial biogenesis and OXPHOS to promote metabolisms (LeBleu et al., 2014). To confirm the decrease in mitochondrial biogenesis in adult IUGR pigs through the SIRT1/PGC1 $\alpha$ pathway, we assessed the activity of the PGC1 $\alpha / \mathrm{NRF} 1 / \mathrm{mtTFA}$ pathway. It was observed that the expression of NRF1 and $m t T F A$ were both decreased in IUGR livers (Figure 5B). It has been well established that NRF1 and mtTFA transactivate the promoters of a number of mitochondrial-related genes and up-regulate mtDNA contents (May-Panloup et al., 2005). To further verify the decrease in 
mtDNA in adult IUGR pigs (Figure 1E), we measured randomly selected mitochondrial coding genes (Shen et al., 2016). As presented in Figure 5C, the expressions of ND3, COX2, and ATP6 mRNAs were significantly decreased in livers of adult IUGR pigs $(p<0.05)$. These results suggest that the capacity of mitochondrial biogenesis is diminished in livers of adult IUGR pigs. The major mitochondrial function was affecting the efficiency of the electron movement through the electron transport chain and its coupling to OXPHOS to produce ATP. Therefore, to explore whether damaged mitochondrial biogenesis influenced the process of OXPHOS, we assessed the expressions of genes related to electron transport chain complexes (Pospisilik et al., 2007). We observed that most of these genes were down-regulated in IUGR livers (Figure 5D). The low efficiency of the electron transport chain in IUGR pigs resulted in decreased ATP production, which could be inferred through the increase in the AMP/ATP ratio (Figure 5E). The observed effect is consistent with the decreased expressions of ATP synthesis genes (ATP5A1 and ATP5B) in livers of adult IUGR pigs (Figure 5F). These findings are corroborated by a previous report that the capacity of hepatic mitochondrial biogenesis and energy metabolism is decreased in weanling pigs (Su et al., 2017). All these results implied that IUGR would damage hepatic mitochondrial biogenesis and OXPHOS in adult pigs.

Previous studies have reported that dysfunctional mitochondria in IUGR can increase superoxide radical metabolites, which may lead to the development of oxidative stress (Biri et al., 2007). MDA would then accumulate in mitochondria, whereas the production of superoxide radicals increased in mitochondria (Che et al., 2015). The high content of MDA in IUGR livers suggested that adult IUGR livers produced excessive oxidative species (Figure 5G). This is in agreement with a previous report in which the antioxidative abilities in neonatal and adult IUGR mice livers were damaged (Gao et al., 2016). Interestingly, CuZn-SOD and GR were regarded as antioxidant enzymes, which would eliminate reactive oxygen species (ROS) (Yang et al., 2013). Here, the reduced activity of CuZn-SOD and GR in livers of adult IUGR pigs suggested the excessive production of free radicals, exhausting the antioxidant enzymes within the cells (Figures $\mathbf{5 H}, \mathbf{I}$ ). This was consistent with the reduced expressions of $\mathrm{CuZn-SOD}$ and GR mRNA in the IUGR animals (Figure 5J). These data suggested that the adult IUGR body possessed an oxidative status in the liver. This observation is consistent with the findings of a previous report, which showed that adult growth-restricted offsprings would exhibit increased oxidative stress and decreased antioxidant activity in a mouse model (Ojeda et al., 2012). Therefore, all these data indicate a possible mechanism of IUGR influenced hepatic energy metabolism and oxidative stress in adults, as presented in Figure 5K.

\section{REFERENCES}

Ackerman, W., Buhimschi, I. A., Summerfield, T., Zhao, G., Landon, M. B., and Buhimschi, C. S. (2017). 222: global transcriptomic analysis of human placenta

\section{CONCLUSION}

In summary, this study provided a transcriptomic analysis between adult normal and IUGR pigs. We identified numerous DEGs, which are potentially associated with oxidoreductase activity, ATPase activity, amino catabolic processes, type 2 diabetes mellitus, and insulin signaling pathways. The expression pattern of DEGs suggested that adult IUGR pigs exhibited strong gluconeogenic activities but a low capacity for fatty acid oxidation, mitochondrial biogenesis, and OXPHOS. This study should serve as a valuable resource for further study of IUGR metabolic syndrome.

\section{ACCESSION CODES}

All the high-throughput sequencing data have been deposited in NCBI's Gene Expression Omnibus under GEO Series accession numbers GSE106512.

\section{AUTHOR CONTRIBUTIONS}

LS, SZ, and LZ conceived and designed the experiments and drafted the manuscript. ML and LS performed the data analysis. SZ, JM, YJ, ML, JW, XL, LC, and GT collected the samples, performed the statistical analysis, and prepared nucleic acids. All authors read and approved the final manuscript.

\section{FUNDING}

We are grateful to Animal Nutrition Institute of Sichuan Agricultural University for animal feeding. This study was supported by the Sichuan Science and Technology Support Program (Pig Genetic Resources Exploitation and Utilization for the 13th Five-Year-Project, No. 16ZC2838) the Chinese National Science and Technology Support Program (No. 2015BAD03B01), the earmarked fund for China Agriculture Research System (No. CARS-36-05B). This work was also partially supported by the China Scholarship Council (CSC) under the Ph.D. visiting student exchange program.

\section{SUPPLEMENTARY MATERIAL}

The Supplementary Material for this article can be found online at: https://www.frontiersin.org/articles/10.3389/fgene. 2018.00291/full\#supplementary-material

in the setting of intrauterine growth restriction (IUGR) using RNA sequencing (RNA-Seq). Am. J. Obstet. Gynecol. 216:S140. doi: 10.1016/j.ajog.2016.11.127

Biri, A., Bozkurt, N., Turp, A., Kavutcu, M., Himmetoglu, Ö, and Durak, Ý (2007). Role of oxidative stress in intrauterine growth 
restriction. Gynecol. Obstet. Invest. 64, 187-192. doi: 10.1159/00010 6488

Bogdarina, I., Murphy, H. C., Burns, S. P., and Clark, A. J. (2004). Investigation of the role of epigenetic modification of the rat glucokinase gene in fetal programming. Life Sci. 74, 1407-1415. doi: 10.1016/j.lfs.2003.08.017

Cantó, C., and Auwerx, J. (2009). PGC-1alpha, SIRT1 and AMPK, an energy sensing network that controls energy expenditure. Curr. Opin. Lipidol. 20, 98-105. doi: 10.1097/MOL.0b013e328328d0a4

Che, L., Thymann, T., Bering, S. B., Le Huërou-Luron, I., D'inca, R., Zhang, K., et al. (2010). IUGR does not predispose to necrotizing enterocolitis or compromise postnatal intestinal adaptation in preterm pigs. Pediatr. Res. 67, 54-59. doi: 10.1203/PDR.0b013e3181c1b15e

Che, L., Xuan, Y., Hu, L., Liu, Y., Xu, Q., Fang, Z., et al. (2015). Effect of postnatal nutrition restriction on the oxidative status of neonates with intrauterine growth restriction in a pig model. Neonatology 107, 93-99. doi: 10.1159/ 000368179

Cheng, Z., and Ristow, M. (2013). Mitochondria and metabolic homeostasis. Antioxid Redox Signal. 19, 240-242. doi: 10.1089/ars.2013.5255

Collier, J. J., and Scott, D. K. (2004). Sweet changes: glucose homeostasis can be altered by manipulating genes controlling hepatic glucose metabolism. Mol. Endocrinol. 18, 1051-1063. doi: 10.1210/me.2003-0357

Egan, A., MacRae, J., and Lamb, C. (1983). Threonine metabolism in sheep: 1. Threonine catabolism and gluconeogenesis in mature blackface wethers given poor quality hill herbage. Br. J. Nutr. 49, 373-383. doi: 10.1079/BJN198 30046

Fernandez-Twinn, D., Wayman, A., Ekizoglou, S., Martin, M., Hales, C., and Ozanne, S. (2005). Maternal protein restriction leads to hyperinsulinemia and reduced insulin-signaling protein expression in 21-mo-old female rat offspring. Am. J. Physiol. Regul. Integr. Comp. Physiol. 288, R368-R373. doi: 10.1152/ ajpregu.00206.2004

Gao, L., Yu, H., Fan, J., Li, L., and Xiao, R. (2016). Effects of maternal high-fat diets during pregnancy and gestation with phytosterols supplementation on oxidative status in neonate, sucking and adult offspring. Obese Open Access 2. doi: 10.16966/2380-5528.119

George, L. A., Zhang, L., Tuersunjiang, N., Ma, Y., Long, N. M., Uthlaut, A. B., et al. (2012). Early maternal undernutrition programs increased feed intake, altered glucose metabolism and insulin secretion, and liver function in aged female offspring. Am. J. Physiol. Regul. Integr. Comp. Physiol. 302, R795-R804. doi: 10.1152/ajpregu.00241.2011

Harmayani, E., Lestari, L. A., Sari, P. M., and Gardjito, M. (2017). "Local Food Diversification and Its (Sustainability) Challenges," in Sustainability Challenges in the Agrofood Sector, ed. R. Bhat (Hoboken, NJ:John Wiley \& Sons Ltd), 119-149. doi: 10.1126/sciadv. 1500558

Hock, M. B., and Kralli, A. (2009). Transcriptional control of mitochondrial biogenesis and function. Annu. Rev. Physiol. 71, 177-203. doi: 10.1146/annurev. physiol.010908.163119

Jitrapakdee, S. (2012). Transcription factors and coactivators controlling nutrient and hormonal regulation of hepatic gluconeogenesis. Int. J. Biochem. Cell Biol. 44, 33-45. doi: 10.1016/j.biocel.2011.10.001

Karpe, F., Dickmann, J. R., and Frayn, K. N. (2011). Fatty acids, obesity, and insulin resistance: time for a reevaluation. Diabetes Metab. Res. Rev. 60, 2441-2449. doi: $10.2337 / \mathrm{db} 11-0425$

Kim, J. K., Zisman, A., Fillmore, J. J., Peroni, O. D., Kotani, K., Perret, P., et al. (2001). Glucose toxicity and the development of diabetes in mice with musclespecific inactivation of GLUT4. J. Clin. Invest. 108, 153-160. doi: 10.1172/ JCI10294

Koklu, E., Kurtoglu, S., Akcakus, M., Koklu, S., Buyukkayhan, D., Gumus, H., et al. (2006). Increased aortic intima-media thickness is related to lipid profile in newborns with intrauterine growth restriction. Horm. Res. 65, 269-275. doi: $10.1159 / 000092536$

Krook, A., Björnholm, M., Galuska, D., Jiang, X. J., Fahlman, R., Myers, M. G., et al. (2000). Characterization of signal transduction and glucose transport in skeletal muscle from type 2 diabetic patients. Diabetes Metab. Res. Rev. 49, 284-292. doi: 10.2337/diabetes.49.2.284

Lagouge, M., Argmann, C., Gerhart-Hines, Z., Meziane, H., Lerin, C., Daussin, F., et al. (2006). Resveratrol improves mitochondrial function and protects against metabolic disease by activating SIRT1 and PGC-1 $\alpha$. Cell 127, 1109-1122. doi: 10.1016/j.cell.2006.11.013
LeBleu, V. S., O'Connell, J. T., Herrera, K. N. G., Wikman-Kocher, H., Pantel, K., Haigis, M. C., et al. (2014). PGC-1 $\alpha$ mediates mitochondrial biogenesis and oxidative phosphorylation to promote metastasis. Nat. Cell Biol. 16, 992-1003. doi: $10.1038 / \mathrm{ncb} 3039$

Leturque, A., Loizeau, M., Vaulont, S., Salminen, M., and Girard, J. (1996). Improvement of insulin action in diabetic transgenic mice selectively overexpressing GLUT4 in skeletal muscle. Diabetes Metab. Res. Rev. 45, 23-27. doi: $10.2337 /$ diab.45.1.23

Li, M., Wu, H., Luo, Z., Xia, Y., Guan, J., Wang, T., et al. (2012). An atlas of DNA methylomes in porcine adipose and muscle tissues. Nat. Commun. 3:850. doi: $10.1038 /$ ncomms 1854

Li, W., Li, B., Lv, J., Dong, L., Zhang, L., and Wang, T. (2016). Choline supplementation improves the lipid metabolism of intrauterine-growthrestricted pigs. Asian Australas J. Anim. Sci. 31, 686-695. doi: 10.5713/ajas.15. 0810

Liu, J., Chen, D., Yao, Y., Yu, B., Mao, X., He, J., et al. (2012). Intrauterine growth retardation increases the susceptibility of pigs to high-fat diet-induced mitochondrial dysfunction in skeletal muscle. PLoS One 7:e34835. doi: 10.1371/ journal.pone. 0034835

Lopez, S., Bermudez, B., Ortega, A., Varela, L. M., Pacheco, Y. M., Villar, J., et al. (2011). Effects of meals rich in either monounsaturated or saturated fat on lipid concentrations and on insulin secretion and action in subjects with high fasting triglyceride concentrations. Am. J. Clin. Nutr. 93, 494-499. doi: 10.3945/ajcn. 110.003251

Manten, G. T., Sikkema, M. J., Voorbij, H. A., Visser, G. H., Bruinse, H. W., and Franx, A. (2007). Risk factors for cardiovascular disease in women with a history of pregnancy complicated by preeclampsia or intrauterine growth restriction. Hypertens. Pregnancy 26, 39-50. doi: 10.1080/10641950601146574

Martin-Gronert, M., and Ozanne, S. E. (2007). Experimental IUGR and later diabetes. J. Intern. Med. 261, 437-452. doi: 10.1111/j.1365-2796.2007.01 800.x

May-Panloup, P., Vignon, X., Chrétien, M.-F., Heyman, Y., Tamassia, M., Malthièry, Y., et al. (2005). Increase of mitochondrial DNA content and transcripts in early bovine embryogenesis associated with upregulation of mtTFA and NRF1 transcription factors. Reprod. Biol. Endocrinol. 3:65. doi: 10.1186/1477-7827-3-65

McMillen, I. C., and Robinson, J. S. (2005). Developmental origins of the metabolic syndrome: prediction, plasticity, and programming. Physiol. Rev. 85, 571-633. doi: 10.1152/physrev.00053.2003

Mortensen, O. H., Olsen, H. L., Frandsen, L., Nielsen, P. E., Nielsen, F. C., Grunnet, N., et al. (2010). A maternal low protein diet has pronounced effects on mitochondrial gene expression in offspring liver and skeletal muscle; protective effect of taurine. J. Biomed. Sci. 17:S38. doi: 10.1186/1423-0127-17S1-S38

Mutz, K.-O., Heilkenbrinker, A., Lönne, M., Walter, J.-G., and Stahl, F. (2013). Transcriptome analysis using next-generation sequencing. Curr. Opin. Biotechnol. 24, 22-30. doi: 10.1016/j.copbio.2012.09.004

Nathan, D. M., Buse, J. B., Davidson, M. B., Ferrannini, E., Holman, R. R., Sherwin, R., et al. (2009). Medical management of hyperglycemia in type 2 diabetes: a consensus algorithm for the initiation and adjustment of therapy. Diabetes Care 32, 193-203. doi: 10.2337/dc08-9025

Nebendahl, C., Krüger, R., Görs, S., Albrecht, E., Martens, K., Hennig, S., et al. (2013). Effects on transcriptional regulation and lipid droplet characteristics in the liver of female juvenile pigs after early postnatal feed restriction and refeeding are dependent on birth weight. PLoS One 8:e76705. doi: 10.1371/ journal.pone. 0076705

Ojeda, N. B., Hennington, B. S., Williamson, D. T., Hill, M. L., Betson, N. E., Sartori-Valinotti, J. C., et al. (2012). Oxidative stress contributes to sex differences in blood pressure in adult growth-restricted offspringnovelty and significance. Hypertension 60, 114-122. doi: 10.1161/HYPERTENSIONAHA. 112.192955

Pallotto, E. K., and Kilbride, H. W. (2006). Perinatal outcome and later implications of intrauterine growth restriction. Clin. Obstet. Gynecol. 49, 257-269. doi: 10. 1097/00003081-200606000-00008

Pospisilik, J. A., Knauf, C., Joza, N., Benit, P., Orthofer, M., Cani, P. D., et al. (2007). Targeted deletion of AIF decreases mitochondrial oxidative phosphorylation and protects from obesity and diabetes. Cell 131, 476-491. doi: 10.1016/j.cell. 2007.08.047 
Ramayo-Caldas, Y., Mach, N., Esteve-Codina, A., Corominas, J., Castelló, A., Ballester, M., et al. (2012). Liver transcriptome profile in pigs with extreme phenotypes of intramuscular fatty acid composition. BMC Genomics 13:547. doi: 10.1186/1471-2164-13-547

Rinaudo, P., and Wang, E. (2012). Fetal programming and metabolic syndrome. Annu. Rev. Physiol. 74, 107-130. doi: 10.1146/annurev-physiol-020911-153245

Rommel, C., Bodine, S. C., Clarke, B. A., Rossman, R., Nunez, L., Stitt, T. N., et al. (2001). Mediation of IGF-1-induced skeletal myotube hypertrophy by PI (3) K/Akt/mTOR and PI (3) K/Akt/GSK3 pathways. Nat. Cell Biol. 3, 1009-1013. doi: 10.1038/ncb1101-1009

Ross, M. G., and Beall, M. H. (2008). Adult sequelae of intrauterine growth restriction. Semin. Perinatol. 32, 213-218. doi: 10.1053/j.semperi.2007.11.00

Sakamaki, J.-I., Daitoku, H., Kaneko, Y., Hagiwara, A., Ueno, K., and Fukamizu, A. (2012). GSK3 $\beta$ regulates gluconeogenic gene expression through $\mathrm{HNF} 4 \alpha$ and FOXO1. J. Recept. Signal. Transduct. Res. 32, 96-101. doi: 10.3109/10799893. 2012.660531

Saltiel, A. R., and Kahn, C. R. (2001). Insulin signalling and the regulation of glucose and lipid metabolism. Nature 414, 799-806. doi: 10.1038/414799a

Samuel, V. T., and Shulman, G. I. (2012). Mechanisms for insulin resistance: common threads and missing links. Cell 148, 852-871. doi: 10.1016/j.cell.2012. 02.017

Sandoval, I. V., and Sols, A. (1974). Gluconeogenesis from serine by the serinedehydratase-dependent pathway in rat liver. FEBS J. 43, 609-616. doi: 10.1111/ j.1432-1033.1974.tb03448.x

Seubert, W., and Huth, W. (1965). On the mechanism of gluconeogenesis and its regulation. II. The mechanism of gluconeogenesis from pyruvate and fumarate. Biochem. Z 34, 176-191.

Shen, L., Luo, J., Du, J., Liu, C., Wu, X., Pu, Q., et al. (2015). Transcriptome analysis of liangshan pig muscle development at the growth curve inflection point and asymptotic stages using digital gene expression profiling. PLoS One 10:e0135978. doi: 10.1371/journal.pone.0135978

Shen, L.-Y., Liu, C.-D., Zhang, S.-H., and Zhu, L. (2016). The complete sequence of the mitochondrial genome of Liangshan pig (Sus Scrofa). Mitochondrial DNA A 27, 4183-4184. doi: 10.3109/19401736.2014.1003903

Snel, M., Jonker, J. T., Schoones, J., Lamb, H., de Roos, A., Pijl, H., et al. (2012). Ectopic fat and insulin resistance: pathophysiology and effect of diet and lifestyle interventions. Int. J. Endocrinol. 2012:983814. doi: 10.1155/2012/ 983814

Su, W., Xu, W., Zhang, H., Ying, Z., Zhou, L., Zhang, L., et al. (2017). Effects of dietary leucine supplementation on the hepatic mitochondrial biogenesis and energy metabolism in normal birth weight and intrauterine growth-retarded weanling piglets. Nutr. Res. Pract. 11, 121-129. doi: 10.4162/nrp.2017.11.2.121

Tain, Y.-L., Huang, L.-T., Chan, J. Y., and Lee, C.-T. (2015). Transcriptome analysis in rat kidneys: importance of genes involved in programmed hypertension. Int. J. Mol. Sci. 16, 4744-4758. doi: 10.3390/ijms16034744

Thorn, S. R., Brown, L. D., Rozance, P. J., Hay, W. W., and Friedman, J. E. (2013). Increased hepatic glucose production in fetal sheep with intrauterine growth restriction is not suppressed by insulin. Diabetes 62 , 65-73. doi: $10.2337 / \mathrm{db} 11$ 1727

Thorn, S. R., Regnault, T. R., Brown, L. D., Rozance, P. J., Keng, J., Roper, M., et al. (2009). Intrauterine growth restriction increases fetal hepatic gluconeogenic capacity and reduces messenger ribonucleic acid translation initiation and nutrient sensing in fetal liver and skeletal muscle. Endocrinology 150, 3021-3030. doi: 10.1210/en.2008-1789

Thorn, S. R., Rozance, P. J., Brown, L. D., and Hay, W. W. (2011). The intrauterine growth restriction phenotype: fetal adaptations and potential implications for later life insulin resistance and diabetes. Semin. Reprod. Med. 29, 225-236. doi: 10.1055/s-0031-1275516

Verma, P., and Chaudhary, H. (2016). Understanding intrauterine growth restriction (IUGR): a review. J. Biomed. Sci. 2, 31-37. doi: 10.3126/jbs.v2i4. 15426

Wang, J., Cao, M., Zhuo, Y., Che, L., Fang, Z., Xu, S., et al. (2016). Catch-up growth following food restriction exacerbates adulthood glucose intolerance in pigs exposed to intrauterine undernutrition. Nutrition 32, 1275-1284. doi: 10.1016/j.nut.2016.03.010

Wolfrum, C., Besser, D., Luca, E., and Stoffel, M. (2003). Insulin regulates the activity of forkhead transcription factor Hnf-3 $\beta$ /Foxa- 2 by Akt-mediated phosphorylation and nuclear/cytosolic localization. Proc. Natl. Acad. Sci. U.S.A. 100, 11624-11629. doi: 10.1073/pnas.1931483100

Yan, H., Zheng, P., Yu, B., Yu, J., Mao, X., He, J., et al. (2017). Postnatal high-fat diet enhances ectopic fat deposition in pigs with intrauterine growth retardation. Eur. J. Nutr. 56, 483-490. doi: 10.1007/s00394-0151093-9

Yang, J., Dong, S., Jiang, Q., Si, Q., Liu, X., and Yang, J. (2013). Characterization and expression of cytoplasmic copper/zinc superoxide dismutase (CuZn SOD) gene under temperature and hydrogen peroxide $\left(\mathrm{H}_{2} \mathrm{O}_{2}\right)$ in rotifer Brachionus calyciflorus. Gene 518, 388-396. doi: 10.1016/j.gene.2012. 12.101

Zhang, H., Li, Y., Hou, X., Zhang, L., and Wang, T. (2016). Medium-chain TAG improve energy metabolism and mitochondrial biogenesis in the liver of intrauterine growth-retarded and normal-birth-weight weanling piglets. Br. J. Nutr. 115, 1521-1530. doi: 10.1017/S0007114516000404

Zhang, H., Li, Y., Su, W., Ying, Z., Zhou, L., Zhang, L., et al. (2017). Resveratrol attenuates mitochondrial dysfunction in the liver of intrauterine growth retarded suckling piglets by improving mitochondrial biogenesis and redox status. Mol. Nutr. Food. Res. 61:1600653. doi: 10.1002/mnfr.201600653

Zhang, W., Patil, S., Chauhan, B., Guo, S., Powell, D. R., Le, J., et al. (2006). FoxO1 regulates multiple metabolic pathways in the liver effects on gluconeogenic, glycolytic, and lipogenic gene expression. J. Biol. Chem. 281, 10105-10117. doi: 10.1074/jbc.M600272200

Conflict of Interest Statement: The authors declare that the research was conducted in the absence of any commercial or financial relationships that could be construed as a potential conflict of interest.

Copyright (C) 2018 Shen, Gan, Zhang, Ma, Tang, Jiang, Li, Wang, Li, Che and Zhu. This is an open-access article distributed under the terms of the Creative Commons Attribution License (CC BY). The use, distribution or reproduction in other forums is permitted, provided the original author(s) and the copyright owner(s) are credited and that the original publication in this journal is cited, in accordance with accepted academic practice. No use, distribution or reproduction is permitted which does not comply with these terms. 